\title{
A constitutive equation for non-linear electro-active solids
}

\author{
K. R. Rajagopal, College Station, Texas, and A. Wineman, Ann Arbor, Michigan
}

(Received December 8, 1996; revised December 2, 1997)

Summary. Electro-active solids are solids that are either infused with electrorheological fluids or embedded with electrically conducting particles, the body as a whole however conducting negligible current. In this paper, we provide a mathematical framework, within the context of continuum mechanics, for the study of electro-active solids. The theory assumes that the body can be considered as a continuum, in the sense of homogenization, which is isotropic, incompressible, elastic and is capable of responding to an electric field. Appealing to standard techniques in continuum mechanics, we obtain a constitutive relation for the stresses in terms of the deformation and electric field. This is used in a study of triaxial extension, simple shear and anisotropy induced by the electric field.

\section{Introduction}

Electro-active solids are solids that are either infused with electrorheological fluids or embedded with electrically conducting particles, the body as a whole however conducting negligible current. Such materials find a variety of applications in hydroacoustics, vibration damping, controllers, actuators, valves, seals and sensing devices to name but a few. In addition to short response times of the order of milliseconds, such electro-active solids have the added advantage of being highly flexible and are amenable to being manufactured into conformable shapes and structures. Recently, there has been a concerted effort to gainfully exploit the potential of such materials. However, much of this work has been devoted to the manufacture, design and engineering of the material with the desired microstructural features, with little if any effort being expended in deriving a reliable mathematical model based on the basic principles of physics that can describe the heretofore observed response of such materials, and even more so possess a modicum of predictive capability.

In this paper, we provide a mathematical framework, within the context of continuum mechanics, for the study of electro-active solids. The theory assumes that the body can be considered as a continuum in the sense of homogenization. We shall restrict our analysis to the domain of mechanics, ignoring completely thermodynamic issues. The electro-active solid will be assumed to be an isotropic incompressible elastic solid, and even with the inclusion of the electrorheological fluid or the embedded particles, it will be treated a though it is a single elastic continuum that is capable of responding to an electric field.

A proper study of the mechanics of such a body would require us to consider an electromechanical framework similar to the theory due to Rajagopal and Ruzicka [1] for electrorheological fluids, i.e. a full set of field equations including Maxwell's equations. Such a system leads to a set of thirteen coupled partial differential equations and the study of initial/ 
boundary value problems in even simple domains becomes most cumbersome. In fact, Toupin [2] developed a general framework for the study of elastic dielectrics, with polarization playing a central role. Here, we would like to provide a simplification that allows us to capture much of the interesting interplay between the electric field and the mechanical dissipation without having to reckon with the full set of equations. Such a simplified theory can be used in more complex geometries that relate to realistic problems. In fact, this approach is quite similar to that adopted for the study of electrorheological fluids by Rajagopal and Wineman [3] that has been able to predict the presence of induced normal stresses due to the electric field and other associated phenomena. In this sense, the study carried herein is the analog of the earlier work on electrorheological fluids to solids. Bearing this in mind, we simplify the problem by treating the electric field as a parameter in the theory rather than a field variable, much in the spirit of the theories constructed by Rajagopal and Wineman [3] and Wineman and Rajagopal [4] for electrorheological fluids, the virtue of such an approximation being validated or otherwise by its predictions.

Using invariant-theoretical considerations presented in the review article by Spencer [5], we obtain constitutive relations to describe the stresses that develop in a deformed solid due to the presence of an electric field. We then study two simple deformations within the context of our theory, the problems of triaxial extension and shear, both providing an opportunity for the evaluation of the theory. The main advantages of the proposed theory are that it is presented within a full three dimensional context that takes into account the requirements of frame-indifference, and allows the study of bodies with different material symmetries.

This approach has been used to study a variety of phenomena in non-linear continuum physics. Pipkin and Rivlin used it to discuss electrical or thermal conduction in deformed isotropic materials [6], galvanomagnetic and thermomagnetic effects in isotropic materials [7], and magnetic hysteresis [8]. Toupin and Rivlin considered electro-magneto-optical effects in isotropic materials [9]. (Indeed, Pipkin and Rivlin state in [6] that "Experience has shown that if an effect is allowed by material symmetry, then it can eventually be produced experimentally in a suitable material unless thermodynamic restrictions rule out the possibility of such behavior".) Thus, the approach in the present paper, just as in the work just cited, is to point out phenomena that can reasonably be expected to occur in electro-active solids.

The arrangement of the paper is as follows. In Section 2, we discuss the basic constitutive theory and provide a representation for the stress based on the work of Spencer [5]. This is followed in Section 3 by a study of triaxial extension of a cube of an electro-active solid where it is shown that a stress free state in the presence of an electric field is a strained state. In Section 4 an analysis of simple shear in the presence of an electric field oriented at an angle to the plane of shear is carried out wherein it is shown that the absence of a shear strain but the presence of an electric field produces both shear and normal stresses. In Section 5, we present a discussion of anisotropy induced by the electric field. Section 6 is devoted to a discussion of the response when the electric field is applied along a principal direction of stretch, which does not coincide with a coordinate axis. A number of issues associated with the representation of the constitutive equation are considered in Section 7.

\section{Constitutive relations}

As we remarked in the Introduction, we are concerned with the mechanics of solids that are either infused with an electrorheological fluid or embedded with particles that respond to the presence of an electric field, with the caveat that the particles of the electrorheological fluid do 
not move relative to the solid ${ }^{1}$. An additional assumption is that this mixture of the solid matrix and the embedded fluid particles/electrorheological fluid can be considered in a homogenized sense as a single continuum, referred to as an electrorheological solid. We shall assume that on the application of an external electric field this continuum responds instantaneously, based on our experience garnered within the context of electrorheological fluids. Furthermore, as a first approximation it seems reasonable to ignore long range effects (nonlocal effects) and the viscoelastic properties of the solid matrix. The analysis of Rajagopal and Wineman [4] to incorporate viscoelastic effects in electrorheological fluids can be easily extended to electro-active solids which are viscoelastic. We refer the reader to [4] for a detailed discussion of the modeling. Also, as is customary in modeling the behavior of electrorheological fluids, we shall suppose that the electric field is essentially constant in the solid, though it can vary with time, and is not determined by solving Maxwell's equation (see [1]). Such an assumption would be particularly appropriate when we are interested in studying inhomogeneous deformations of the solid.

It would be reasonable under the above assumptions to assume that the stress at any point in the electro-active solid depends on the deformation gradient $\mathbf{F}$ and the electric field vector $\mathbf{E}$, the latter playing the role of a parameter rather than an unknown field ${ }^{2}$. Thus, the Cauchy stress $\mathbf{T}$ is given by

$\mathbf{T}=\mathbf{f}(\varrho, \mathbf{F}, \mathbf{E})$,

where $\varrho$ denotes the density and

$\mathbf{F}:=\frac{\partial \chi}{\partial \mathbf{X}}$

where

$\mathbf{x}=\chi(\mathbf{X}, t)$

denotes the motion of the body. Although it is possible to construct electro-active solids with foams, we shall be primarily concerned with those solids which are incompressible. Such solids can only undergo motions that meet

$\operatorname{det} \mathbf{F}=1$

and the stress $\mathbf{T}$ in such materials is determined only up to an arbitrary spherical part $-p \mathbf{I}$,

$\mathbf{T}=-p \mathbf{I}+\mathbf{g}(\mathbf{F}, \mathbf{E})$.

It follows from frame-indifference that $\mathrm{g}$ must satisfy

$\mathbf{g}(\mathbf{Q F}, \mathbf{Q E})=\mathbf{Q g}(\mathbf{F}, \mathbf{E}) \mathbf{Q}^{T}$

for all $\mathbf{Q}$ belonging to the proper orthogonal group, i.e. $\operatorname{det} \mathbf{Q}=1$. A consequence of Eq. (6) is that $\mathbf{g}$ has to satisfy

$\mathbf{g}(\mathbf{F}, \mathbf{E})=\mathbf{R} \tilde{\mathbf{g}}\left(\mathbf{U}, \mathbf{F}^{T} \mathbf{E}\right) \mathbf{R}^{T}$,

\footnotetext{
${ }^{1}$ If such relative motion is to be taken into account, then we would have to appeal to a theory such as the theory of interacting continua (see Rajagopal and Wineman [10], Rajagopal and Tao [11]).

${ }^{2}$ The model that is proposed would also be valid for viscoelastic materials provided that we are interested in the long term steady response of the electro-active solid in the presence of an electric field, especially for DC voltages.
} 
where $\mathbf{F}=\mathbf{R} \mathbf{U}$ and $\mathbf{R}$ is a rigid body rotation. A further simplification leads to

$\mathbf{g}(\mathbf{F}, \mathbf{E})=\mathbf{F} \hat{\mathbf{g}}\left(\mathbf{C}, \mathbf{F}^{T} \mathbf{E}\right) \mathbf{F}^{T}$,

where $\mathbf{C}=\mathbf{F}^{T} \mathbf{F}$. If the symmetry group at a point in the body is $\mathcal{G}$, then $\hat{\mathbf{g}}$ has to satisfy the additional restriction

$\hat{\mathbf{g}}\left(\mathbf{H}^{T} \mathbf{C H}, \mathbf{H}^{T} \mathbf{F}^{T} \mathbf{E}\right)=\mathbf{H}^{T} \hat{\mathbf{g}}\left(\mathbf{C}, \mathbf{F}^{T} \mathbf{E}\right) \mathbf{H}$,

for $\mathbf{H} \in \mathcal{G}$.

We next discuss issues regarding the material symmetry of the body. If the distribution of the embedded electrically responding medium is homogeneous and if the solid is isotropic, we can assume that the electro-active solid is also isotropic. There has been some discussion as to whether the symmetry group $\mathcal{G}$ should be the full orthogonal group or just the proper orthogonal sub-group. Here we shall document the representation of $\hat{g}$ for both cases.

In the case when $\mathcal{G}$ is the full orthogonal group, we have the following representation for $\hat{\mathrm{g}}$ (see Spencer [5] $)^{3}$ :

$$
\begin{aligned}
\mathbf{T}= & -p \mathbf{I}+\alpha_{1} \mathbf{E} \otimes \mathbf{E}+\alpha_{2} \mathbf{B}+\alpha_{3} \mathbf{B}^{-1}+\alpha_{4}(\mathbf{B E} \otimes \mathbf{E}+\mathbf{E} \otimes \mathbf{B E}) \\
& +\alpha_{5}\left(\mathbf{B}^{-1} \mathbf{E} \otimes \mathbf{E}+\mathbf{E} \otimes \mathbf{B}^{-1} \mathbf{E}\right),
\end{aligned}
$$

where $\mathbf{B}=\mathbf{F F}^{T}$, and $\alpha_{i}, i=1, \ldots, 5$ are scalar functions that depend on the following invariants:

$I_{1}=\operatorname{tr}(\mathbf{E} \otimes \mathbf{E}), \quad I_{2}=\operatorname{tr} \mathbf{B}, \quad I_{3}=\frac{1}{2}\left[(\operatorname{tr} \mathbf{B})^{2}-\operatorname{tr} \mathbf{B}^{2}\right]$,

$I_{4}=\operatorname{tr}(\mathbf{B E} \otimes \mathbf{E}), \quad I_{5}=\operatorname{tr}\left(\mathbf{B}^{-1} \mathbf{E} \otimes \mathbf{E}\right)$.

On the other hand, if $\mathcal{G}$ is the proper orthogonal group, then $\hat{\mathrm{g}}$ has the representation

$$
\begin{aligned}
\mathbf{T}= & -p \mathbf{I}+\hat{\alpha}_{1} \mathbf{E} \otimes \mathbf{E}+\hat{\alpha}_{2} \mathbf{B}+\hat{\alpha}_{3} \mathbf{B}^{-1}+\hat{\alpha}_{4}(\mathbf{B E} \otimes \mathbf{E}+\mathbf{E} \otimes \mathbf{B E}) \\
& +\hat{\alpha}_{5}\left(\mathbf{B}^{-1} \mathbf{E} \otimes \mathbf{E}+\mathbf{E} \otimes \mathbf{B}^{-1} \mathbf{E}\right)+\hat{\alpha}_{6}\left(\mathbf{M B}+\mathbf{B M}^{T}\right)+\hat{\alpha}_{7}\left(\mathbf{M B}^{-1}+\mathbf{B}^{-1} \mathbf{M}^{T}\right) \\
& +\hat{\alpha}_{8}\left(\mathbf{B M B}^{-1}+\mathbf{B}^{-1} \mathbf{M}^{T} \mathbf{B}\right)+\hat{\alpha}_{9}\left(\mathbf{M B}^{-1} \mathbf{E} \otimes \mathbf{E}+\mathbf{E} \otimes \mathbf{M B}^{-1} \mathbf{E}\right) \\
& +\hat{\alpha}_{10}(\mathbf{M B} \otimes \mathbf{E}+\mathbf{E} \otimes \mathbf{M B}),
\end{aligned}
$$

where $\hat{\alpha}_{i}, i=1,2, \ldots, 10$ are functions of the following invariants:

$$
\begin{aligned}
& \left.I_{1}=\operatorname{tr}(\mathbf{E} \otimes \mathbf{E}), \quad I_{2}=\operatorname{tr} \mathbf{B}, \quad I_{3}=\operatorname{tr} \mathbf{B}\right)^{-1}, \quad I_{4}=\operatorname{tr}(\mathbf{B E} \otimes \mathbf{E}), \\
& I_{5}=\operatorname{tr}\left(\mathbf{B}^{-1} \mathbf{E} \otimes \mathbf{E}\right), \quad I_{6}=\operatorname{tr}\left(\mathbf{B}^{-1} \mathbf{M}^{T} \mathbf{B}^{-1} \mathbf{E} \otimes \mathbf{E}\right)-\operatorname{tr}\left(\mathbf{B}^{-1} \mathbf{M}^{T} \mathbf{B}^{-1}\right) I_{1} .
\end{aligned}
$$

In Eq. (13), $\mathbf{M}:=\varepsilon \mathbf{E}$, where $\varepsilon$ is the third order alternator tensor.

In this paper, we shall confine our attention to the model defined by Eq. (10) and Eq. (11), bearing in mind that even in this case we have to contend with five unknown material functions that appear in the representation.

\footnotetext{
${ }^{3}$ We derive in Section 7 the appropriate representations for the stress that stem from the irreducible representation for $\hat{\mathrm{g}}$, which seemingly has a different structure than Eq. (10). However, as shown in Section 7, Eq. (10) is an acceptable representation for the stress, though not the simplest.
} 


\section{Triaxial extension}

We shall now consider simple deformations in order to glean some insight into the response of an electro-active solid. Let us first consider the problem in which the electric field vector is along the $X_{1}$ axis and the solid undergoes triaxial extension along the coordinate axes. Then $\mathbf{E}=(E, 0,0)$ and the motion from $\left(X_{1}, X_{2}, X_{3}\right)$ to $\left(x_{1}, x_{2}, x_{3}\right)$ is defined by

$x_{1}=\lambda_{1} X_{1}, \quad x_{2}=\lambda_{2} X_{2}, \quad x_{3}=\lambda_{3} X_{3}$,

where $\lambda_{i}, i=1,2,3$, is a constant and is the stretch ratio in the $i$-th direction. The incompressibility condition Eq. (4) implies that

$\lambda_{1} \lambda_{2} \lambda_{3}=1$.

A routine calculation yields

$$
\begin{aligned}
\mathbf{T}= & -p \mathbf{I}+\alpha_{1}\left[\begin{array}{ccc}
E^{2} & 0 & 0 \\
0 & 0 & 0 \\
0 & 0 & 0
\end{array}\right]+\alpha_{2}\left[\begin{array}{ccc}
\lambda_{1}{ }^{2} & 0 & 0 \\
0 & \lambda_{2}{ }^{2} & 0 \\
0 & 0 & \lambda_{3}{ }^{2}
\end{array}\right]+\alpha_{3}\left[\begin{array}{ccc}
\lambda_{1}{ }^{-2} & 0 & 0 \\
0 & \lambda_{2}{ }^{-2} & 0 \\
0 & 0 & \lambda_{3}{ }^{-2}
\end{array}\right] \\
& +2 \alpha_{4}\left[\begin{array}{ccc}
\lambda_{1}{ }^{2} E^{2} & 0 & 0 \\
0 & 0 & 0 \\
0 & 0 & 0
\end{array}\right]+2 \alpha_{5}\left[\begin{array}{ccc}
\lambda_{1}{ }^{-2} E^{2} & 0 & 0 \\
0 & 0 & 0 \\
0 & 0 & 0
\end{array}\right]
\end{aligned}
$$

where the material functions $\alpha_{i}, i=1, \ldots, 5$ depend on the following invariants:

$I_{1}=E^{2}, \quad I_{2}=\lambda_{1}{ }^{2}+\lambda_{2}{ }^{2}+\lambda_{3}{ }^{2}, \quad I_{3}=\lambda_{1}{ }^{-2}+{\lambda_{2}}^{-2}+\lambda_{3}{ }^{-2}, \quad I_{4}=2 \lambda_{1}{ }^{2} E^{2}, \quad I_{5}=2 \lambda_{1}{ }^{-2} E^{2}$.

Now, we restrict attention to a situation wherein all components of the stress tensor $\mathbf{T}$ other than $T_{11}$ are zero. It immediately follows from Eq. (16) that

$$
\left(\alpha_{2}-\frac{\alpha_{3}}{\lambda_{2}{ }^{2} \lambda_{3}{ }^{2}}\right)\left(\lambda_{2}{ }^{2}-\lambda_{3}{ }^{2}\right)=0 \text {. }
$$

Thus, in materials in which $\alpha_{2} \neq \alpha_{3} / \lambda_{2}{ }^{2} \lambda_{3}{ }^{2}$, we would necessarily have $\lambda_{2}{ }^{2}=\lambda_{3}{ }^{2}$, i.e. the stretch ratios in the directions transverse to the direction of $X_{1}$ are equal, a situation akin to that in non-linear elasticity. We also find that the pressure field is given by

$p=\frac{\alpha_{2}}{\lambda_{1}}+\alpha_{3} \lambda_{1}$

and thus

$T_{11}=\alpha_{1} E^{2}+\left(\lambda_{1}^{2}-\frac{1}{\lambda_{1}}\right)\left(\alpha_{2}-\frac{\alpha_{3}}{\lambda_{1}}\right)+2 E^{2}\left(\alpha_{4} \lambda_{1}^{2}+\frac{\alpha_{5}}{\lambda_{1}^{2}}\right)$.

The above expression for $T_{11}$ leads to a rather interesting result. Suppose $T_{11}=0$ and $E \neq 0$. Then, in general, we find that $\lambda_{1} \neq 1$. That is, the body is stress free in the presence of the electric field, but in a state that is deformed with respect to the stress free state in the absence of the electric field. Thus, a cube whose sides are initially of length "a", deforms on the application of the electric field into a parallelepiped whose sides are of length $\left(a \lambda_{1}, a / \sqrt{\lambda_{1}}\right.$, $a / \sqrt{\lambda_{1}}$, ) along the $X_{1}, X_{2}, X_{3}$ directions, respectively. Depending on the material functions 
$\alpha_{i}, i=1, \ldots, 5$ it is possible that $\lambda_{1}>1$ or that $\lambda_{1}<1$, or more importantly, it is possible that more than one set of values of $\left(\lambda_{1}, \lambda_{2}, \lambda_{3}\right)$ is possible for which the body is in a stress free state. Moreover, as Eq. (16) and Eq. (17) are quadratic in the electric field, the above results hold whether $E<0$ or $E>0$.

\section{Simple shear}

Next, we shall consider the electro-active solid subject to a simple shear and an electric field vector $\mathbf{E}$. The shear is defined through

$x_{1}=X_{1}+K X_{2}, \quad x_{2}=X_{2}, \quad x_{3}=X_{3}$,

while the electric field vector $\mathbf{E}$ has the components $\left(E_{1}, E_{2}, 0\right)$. A lengthy but straightforward computation yields

$$
\begin{aligned}
\mathbf{T}= & -p \mathbf{I}+\alpha_{1}\left[\begin{array}{ccc}
E_{1}{ }^{2} & E_{1} E_{2} & 0 \\
E_{1} E_{2} & E_{2}{ }^{2} & 0 \\
0 & 0 & 0
\end{array}\right]+\alpha_{2}\left[\begin{array}{ccc}
1+K^{2} & K & 0 \\
K & 1 & 0 \\
0 & 0 & 1
\end{array}\right]+\alpha_{3}\left[\begin{array}{ccc}
1 & -K & 0 \\
-K & 1+K^{2} & 0 \\
0 & 0 & 1
\end{array}\right] \\
& +\alpha_{4}\left[\begin{array}{ccc}
2 E_{1}{ }^{2}\left(1+K^{2}\right)+2 E_{1} E_{2} K & E_{1} E_{2}\left(2+K^{2}\right)+\left(E_{1}{ }^{2}+E_{2}{ }^{2}\right) K & 0 \\
E_{1} E_{2}\left(2+K^{2}\right)+\left(E_{1}{ }^{2}+E_{2}{ }^{2}\right) K & 2\left(E_{1} E_{2} K+E_{2}{ }^{2}\right) & 0 \\
0 & 0 & 0
\end{array}\right] \\
& +\alpha_{5}\left[\begin{array}{ccc}
2\left(E_{1}{ }^{2}-E_{1} E_{2} K\right) & E_{1} E_{2}\left(2+K^{2}\right)-\left(E_{1}{ }^{2}+E_{2}{ }^{2}\right) K & 0 \\
E_{1} E_{2}\left(2+K^{2}\right)-\left(E_{1}{ }^{2}+E_{2}{ }^{2}\right) K & 2 E_{2}{ }^{2}\left(1+K^{2}\right)-2 E_{1} E_{2} K & 0 \\
0 & 0 & 0
\end{array}\right],
\end{aligned}
$$

and the material functions $\alpha_{i}, i=1, \ldots, 5$ depend on the invariants

$I_{1}=E_{1}^{2}+E_{2}^{2}, \quad I_{2}=I_{3}=3+K^{2}, \quad I_{4}=E_{1}^{2}\left(1+K^{2}\right)+E_{2}^{2}+2 E_{1} E_{2} K$,

$I_{5}=E_{1}^{2}+E_{2}^{2}\left(1+K^{2}\right)-2 E_{1} E_{2} K$.

It follows immediately that

$T_{11}+p=\alpha_{2}\left(1+K^{2}\right)+\alpha_{3}+E_{1}^{2}\left[\alpha_{1}+2 \alpha_{4}\left(1+K^{2}\right)+2 \alpha_{5}\right]+2 E_{1} E_{2}\left(\alpha_{4}-\alpha_{5}\right) K$,

$T_{22}+p=\alpha_{2}+\alpha_{3}\left(1+K^{2}\right)+E_{2}{ }^{2}\left[\alpha_{1}+2 \alpha_{4}+2 \alpha_{5}\left(1+K^{2}\right)\right]+2 E_{1} E_{2}\left(\alpha_{4}-\alpha_{5}\right) K$,

$T_{12}=\left(\alpha_{2}-\alpha_{3}\right) K+\left(E_{1}^{2}+E_{2}^{2}\right)\left(\alpha_{4}-\alpha_{5}\right) K+E_{1} E_{2}\left[\alpha_{1}+\left(\alpha_{4}+\alpha_{5}\right)\left(2+K^{2}\right)\right]$.

A useful alternate form is obtained by expressing the electric field in terms of its magnitude $E_{0}$,

$$
E_{1}=E_{0} \cos \phi, \quad E_{2}=E_{0} \sin \phi .
$$

Then, Eq. (22) can be written as

$$
\begin{aligned}
& T_{11}+p= \alpha_{2}\left(1+K^{2}\right)+\alpha_{3}+E_{0}{ }^{2} \cos ^{2} \phi\left[\alpha_{1}+2 \alpha_{4}\left(1+K^{2}\right)+2 \alpha_{5}\right] \\
&+E_{0}{ }^{2} \sin 2 \phi\left(\alpha_{4}-\alpha_{5}\right) K, \\
& T_{22}+p= \alpha_{2}+\alpha_{3}\left(1+K^{2}\right)+E_{0}{ }^{2} \sin ^{2} \phi\left[\alpha_{1}+2 \alpha_{4}+2 \alpha_{5}\left(1+K^{2}\right)\right] \\
&+E_{0}{ }^{2} \sin 2 \phi\left(\alpha_{4}-\alpha_{5}\right) K, \\
& T_{12}=\left(\alpha_{2}-\alpha_{3}\right) K+E_{0}{ }^{2}\left(\alpha_{4}-\alpha_{5}\right) K+\frac{1}{2} E_{0}{ }^{2} \sin 2 \phi\left[\alpha_{1}+\left(\alpha_{4}+\alpha_{5}\right)\left(2+K^{2}\right)\right] .
\end{aligned}
$$


The invariants in Eq. (26) become

$I_{1}=E_{0}{ }^{2}, \quad I_{2}=I_{3}=3+K^{2}, \quad I_{4}=E_{0}{ }^{2}+E_{0}{ }^{2} \cos ^{2} \phi K^{2}+E_{0}{ }^{2} \sin ^{2} 2 \phi K$,

$I_{5}=E_{0}{ }^{2}+E_{0}^{2} \sin ^{2} \phi K^{2}-E_{o}^{2} \sin 2 \phi K$.

According to Eq. (24),

$T_{11}-T_{22}=\left(\alpha_{2}-\alpha_{3}\right) K^{2}+\left(E_{1}{ }^{2}-E_{2}{ }^{2}\right)\left(\alpha_{1}+2 \alpha_{4}+2 \alpha_{5}\right)+2 K^{2}\left(\alpha_{4} E_{1}{ }^{2}-\alpha_{5} E_{2}{ }^{2}\right)$,

and we observe that we do not have an universal relation similar to that encountered in nonlinear elasticity.

We observe that the expressions in Eq. (24) and Eq. (26) are quadratic in the components of $\mathrm{E}$. Thus, a reversal of the electric field has no influence on the stresses. The terms containing $\alpha_{2}$ and $\alpha_{3}$ on the right hand sides of Eq. (24) and Eq. (26) remain if $E_{0}=0$, and are thus associated with the non-linear elasticity of the solid material. The terms containing $\alpha_{1}, \alpha_{4}$ and $\alpha_{5}$ are introduced when $E_{0} \neq 0$. There is thus a contribution to the normal stresses and shear stress from the electro-active response of the solid. If the electric field is not along a coordinate direction, i.e. $\phi \neq 0^{\circ}$ or $\phi \neq 90^{\circ}$, then the normal stresses and invariants contain a term that is linear in the shear $K$. If the direction of shear is reversed, while the electric field is held fixed, then there is a change in the normal stresses and the shear stress $T_{12}$ is not an odd function of $K$. It is also interesting to note the consequences of the last term in the expression for the shear stress. It is possible that the shear stress $T_{12}$ could be zero for a finite value of the shear $K$. Also, it is possible that when $K=0$ and $E_{0} \neq 0$, the shear stress $T_{12}$ is non-zero. More generally, even in the absence of shear, the presence of an electric field leads to normal and shear stresses in the body.

\section{Anisotropy induced by the electric field}

Let us now consider the case when $E_{1}=0$, and for convenience let $E_{2}$ be replaced by $E$. It follows from Eq. (23) and Eq. (24) that

$T_{11}=-p+\alpha_{2}\left(1+K^{2}\right)+\alpha_{3}$,

$T_{22}=-p+\alpha_{2}+\alpha_{3}\left(1+K^{2}\right)+E^{2}\left[\alpha_{1}+2 \alpha_{4}+2 \alpha_{5}\left(1+K^{2}\right)\right]$,

$T_{12}=\left[\left(\alpha_{2}-\alpha_{3}\right)+E^{2}\left(\alpha_{4}-\alpha_{5}\right)\right] K$,

and

$I_{1}=E^{2}, \quad I_{2}=I_{3}=3+K^{2}, \quad I_{4}=E^{2}, \quad I_{5}=E^{2}\left(1+K^{2}\right)$.

Then $T_{12}$ can be expressed as

$T_{12}=\mu_{12}\left(K^{2}, E^{2}\right) K$

where

$\mu_{12}=\left(\alpha_{2}-\alpha_{3}\right)+E^{2}\left(\alpha_{4}-\alpha_{5}\right)$

is a shear modulus which depends on the shear $K$ and the electric field $E$.

Next consider a deformation of the form

$x_{1}=X_{1}, \quad x_{2}=X_{2}, \quad x_{3}=X_{3}+K X_{2}$, 
and an electric field $\mathbf{E}$ whose components are again $(0, E, 0)$. It follows that

$$
\begin{aligned}
\mathbf{T}= & -p \mathbf{I}+\alpha_{1}\left[\begin{array}{ccc}
0 & 0 & 0 \\
0 & E^{2} & 0 \\
0 & 0 & 0
\end{array}\right]+\alpha_{2}\left[\begin{array}{ccc}
1 & 0 & 0 \\
0 & 1 & K \\
0 & K & 1+K^{2}
\end{array}\right]+\alpha_{3}\left[\begin{array}{ccc}
1 & 0 & 0 \\
0 & 1+K^{2} & -K \\
0 & -K & 1
\end{array}\right] \\
& +\alpha_{4}\left[\begin{array}{ccc}
0 & 0 & 0 \\
0 & 2 E^{2} & E^{2} K \\
0 & E^{2} K & 0
\end{array}\right]+\alpha_{5}\left[\begin{array}{ccc}
0 & 0 & 0 \\
0 & 2 E^{2}\left(1+K^{2}\right) & -E^{2} K \\
0 & -E^{2} K & 0
\end{array}\right]
\end{aligned}
$$

and the invariants are the same as in Eq. (30). We observe that the normal stress component $T_{22}$ is the same as before, while the component $T_{33}$ plays the role of $T_{11}$. Also, $T_{23}$ can be expressed as

$T_{23}=\mu_{23}\left(K^{2}, E^{2}\right) K$,

with

$\mu_{23}\left(K^{2}, E^{2}\right)=\mu_{12}\left(K^{2}, E^{2}\right)$,

as is to be expected.

Finally, consider the deformation

$x_{1}=X_{1}, \quad x_{2}=X_{2}, \quad x_{3}=X_{3}+K X_{1}$,

with the components of the electric field $\mathbf{E}$ still given by $(0, E, 0)$. It then follows that

$$
\begin{aligned}
& \mathbf{T}=-p \mathbf{I}+\alpha_{1}\left[\begin{array}{ccc}
0 & 0 & 0 \\
0 & E^{2} & 0 \\
0 & 0 & 0
\end{array}\right]+\alpha_{2}\left[\begin{array}{ccc}
1 & 0 & K \\
0 & 1 & 0 \\
K & 0 & 1+K^{2}
\end{array}\right]+\alpha_{3}\left[\begin{array}{ccc}
1+K^{2} & 0 & -K \\
0 & 1 & 0 \\
-K & 0 & 1
\end{array}\right] \\
& +\alpha_{4}\left[\begin{array}{ccc}
0 & 0 & 0 \\
0 & 2 E^{2} & 0 \\
0 & 0 & 0
\end{array}\right]+\alpha_{5}\left[\begin{array}{ccc}
0 & 0 & 0 \\
0 & 2 E^{2} & 0 \\
0 & 0 & 0
\end{array}\right]
\end{aligned}
$$

The first four invariants are the same as in Eq. (30), while now $I_{5}=E^{2}$. The stresses are

$T_{11}=-p+\alpha_{2}+\alpha_{3}\left(1+K^{2}\right), \quad T_{33}=-p+\alpha_{2}\left(1+K^{2}\right)+\alpha_{3}, \quad T_{13}=\left(\alpha_{2}-\alpha_{3}\right) K$.

The shear stress can be expressed as

$T_{13}=\mu_{13}\left(K^{2}, E^{2}\right) K$,

where

$\mu_{13}=\alpha_{2}-\alpha_{3} \neq \mu_{12}=\mu_{23}$.

However, when $E=0$, we recover the identity

$\mu_{13}=\mu_{12}=\mu_{23}$,

for an isotropic solid, indicating the role of the applied electric field in inducing anisotropy of response. 


\section{Response to an electric field applied along a principal direction of stretch}

Recall from Section 3 that when the electric field is along a principal direction of stretch, $\mathbf{T}$ can be expressed by Eq. (16). Now consider a transformation from coordinate system $\left(X_{1}\right.$, $\left.X_{2}, X_{3}\right)$ to coordinate system $\left(X_{1}{ }^{\prime}, X_{2}{ }^{\prime}, X_{3}{ }^{\prime}\right)$ by a rotation of angle $\theta$ about the $X_{3}$ axis, so that the direction cosine matrix is given by

$\mathbf{L}=\left[\begin{array}{ccc}\cos \theta & \sin \theta & 0 \\ -\sin \theta & \cos \theta & 0 \\ 0 & 0 & 1\end{array}\right]$

Thus, the electric field vector $\mathbf{E}$ has components

$\mathbf{E}:=\left(E_{1}^{\prime}, E_{2}{ }^{\prime}, 0\right)=(E \cos \theta,-E \sin \theta, 0)$

with respect to the primed system. It can be easily shown that the deformation Eq. (14) can be expressed in the primed system as

$x_{1}^{\prime}=F_{11}^{\prime} X_{1}{ }^{\prime}+F_{12}^{\prime} X_{2}{ }^{\prime}, \quad x_{2}{ }^{\prime}=F_{21}^{\prime} X_{1}^{\prime}+F_{22}^{\prime} X_{2}{ }^{\prime}, \quad x_{3}{ }^{\prime}=\lambda_{3} X_{3}{ }^{\prime}$,

where $F_{i j}^{\prime}$, the components of the deformation gradient with respect to the $x^{\prime}$-axes, are given by

$\left[F_{i j}^{\prime}\right]=\left[\begin{array}{ccc}\lambda_{1} \cos ^{2} \theta+\lambda_{2} \sin ^{2} \theta & \left(\lambda_{2}-\lambda_{1}\right) \sin \theta \cos \theta & 0 \\ \left(\lambda_{2}-\lambda_{1}\right) \sin \theta \cos \theta & \lambda_{1} \sin ^{2} \theta-\lambda_{2} \cos ^{2} \theta & 0 \\ 0 & 0 & \lambda_{3}\end{array}\right]$.

It is unnecessary for the purposes of the following discussion to document the expression for the stress $\mathbf{T}$ in the primed system.

In the $X_{1}-X_{2}$ plane, a square with edges along the $X_{1}-X_{2}$ axes will deform into a rectangle whose edges are along the $X_{1}-X_{2}$ axes. According to Eqs. (45) and (46), a square in the $X_{1}{ }^{\prime}-X_{2}{ }^{\prime}$ plane with edges along the $X_{1}{ }^{\prime}-X_{2}{ }^{\prime}$ axes will deform into a parallelogram whose diagonal will be along the electric field. This in turn implies that if we apply an electric field that is not along a coordinate direction, then an undeformed cube will undergo both a shear deformation and a stretch perpendicular to the plane of shear.

\section{Comments on the representation of the constitutive equation}

Consider $\hat{\mathbf{g}}\left(\mathbf{C}, \mathbf{F}^{T} \mathbf{E}\right)$ in Eq. (8) and the case when $\mathcal{G}$ is the full orthogonal group in Eq. (9). The methods presented in the article by Spencer [5] lead to the following representation for $\hat{\mathrm{g}}\left(\mathbf{C}, \mathbf{F}^{T} \mathbf{E}\right)$ :

$$
\begin{aligned}
\hat{\mathrm{g}}(\mathbf{C}, \mathbf{Y})= & \gamma_{1} \mathbf{I}+\gamma_{2} \mathbf{Y} \otimes \mathbf{Y}+\gamma_{3} \mathbf{C}+\gamma_{4} \mathbf{C}^{2}+\gamma_{5}(\mathbf{C Y} \otimes \mathbf{Y}+\mathbf{Y} \otimes \mathbf{C Y}) \\
& +\gamma_{6}\left(\mathbf{C}^{2} \mathbf{Y} \otimes \mathbf{Y}+\mathbf{Y} \otimes \mathbf{C}^{2} \mathbf{Y}\right),
\end{aligned}
$$

where $\mathbf{Y}=\mathbf{F}^{T} \mathbf{E}$ and $\gamma_{i}, i=1, \ldots, 5$ are scalar functions which depend on the invariants

$$
\begin{aligned}
& I_{1}=\operatorname{tr}(\mathbf{Y} \otimes \mathbf{Y}), \quad I_{2}=\operatorname{tr} \mathbf{C}, \quad I_{3}=\frac{1}{2}\left[(\operatorname{tr} \mathbf{C})^{2}-\operatorname{tr} \mathbf{C}^{2}\right], \\
& I_{4}=\operatorname{tr}(\mathbf{C Y} \otimes \mathbf{Y}), \quad I_{5}=\operatorname{tr}\left(\mathbf{C}^{2} \mathbf{Y} \otimes \mathbf{Y}\right) .
\end{aligned}
$$


When Eq. (47) is substituted into Eq. (8), the result is

$$
\begin{aligned}
\mathbf{g}(\mathbf{F}, \mathbf{E})= & \gamma_{1} \mathbf{B}+\gamma_{2} \mathbf{B E} \otimes \mathbf{B E}+\gamma_{3} \mathbf{B}^{2}+\gamma_{4} \mathbf{B}^{3}+\gamma_{5}\left(\mathbf{B}^{2} \mathbf{E} \otimes \mathbf{B E}+\mathbf{B E} \otimes \mathbf{B}^{2} \mathbf{E}\right) \\
& +\gamma_{6}\left(\mathbf{B}^{3} \mathbf{E} \otimes \mathbf{B E}+\mathbf{B E} \otimes \mathbf{B}^{3} \mathbf{E}\right),
\end{aligned}
$$

and Eq. (48) becomes

$$
\begin{array}{ll}
I_{1}=\operatorname{tr}(\mathbf{B E} \otimes \mathbf{E}), & I_{2}=\operatorname{tr} \mathbf{B}, \quad I_{3}=\frac{1}{2}\left[(\operatorname{tr} \mathbf{B})^{2}-\operatorname{tr} \mathbf{B}^{2}\right], \\
I_{4}=\operatorname{tr}\left(\mathbf{B}^{2} \mathbf{E} \otimes \mathbf{E}\right), & I_{5}=\operatorname{tr}\left(\mathbf{B}^{3} \mathbf{E} \otimes \mathbf{E}\right) .
\end{array}
$$

By use of the Cayley-Hamilton theorem for $\mathbf{B}$, the set of invariants in Eq. (50) can be reduced to the set of invariants in Eq. (11). Use of the Cayley-Hamilton theorem for B in Eq. (49) leads to terms of the form

$\mathbf{B E} \otimes \mathbf{B E}, \quad \mathbf{B}^{2} \mathbf{E} \otimes \mathbf{B E}+\mathbf{B E} \otimes \mathbf{B}^{2} \mathbf{E}$.

According to the method and results presented by Spencer [5], each tensor in Eq. (51) can be expressed as a sum of terms, each of which is the product of some of the invariants in Eq. (11) and coefficient tensors of the form

$\mathbf{B E} \otimes \mathbf{E}+\mathbf{E} \otimes \mathbf{B E}, \quad \mathbf{B}^{2} \mathbf{E} \otimes \mathbf{E}+\mathbf{E} \otimes \mathbf{B}^{2} \mathbf{E}$.

In this way, the representation of the constitutive equation in Eq. (10) is seen to be one of a number of equivalent possible choices.

\section{References}

[1] Rajagopal, K. R., Ruzicka, M.: Mathematical modeling of electrorheological materials. Int. J. Eng. Sci. (submitted).

[2] Toupin, R. A.: The elastic dielectric. J. Rat. Mech. Anal. 5, 849-915 (1956).

[3] Rajagopal, K. R., Wineman, A. S.: Flow of electrorheological fluids. Acta Mech. 91, 57-75 (1992).

[4] Wineman, A. S., Rajagopal, K. R.: On constitutive equations for electrorheological materials. Cont. Mech. Thermodyn. 7, 1-22 (1995).

[5] Spencer, A. J. M.: Continuum physics (Eringen, A. C., ed.), Vol. 3. New York: Academic Press 1975.

[6] Pipkin, A. C., Rivlin, R. S.: Electrical conduction in deformed isotropic materials. J. Math. Phys. 1, $127-130$ (1960).

[7] Pipkin, A. C., Rivlin, R. S.: Galvanomagnetic and thermomagnetic effects in isotropic materials. J. Math. Phys. 1, 542-546 (1960).

[8] Pipkin, A. C., Rivlin, R. S.: Phenomenological theory of magnetic hysteresis. J. Math. Phys. 8, $878-883$ (1967).

[9] Toupin, R. A., Rivlin, R. S.: Electro-magnetic-optical effects. Arch. Rat. Mech. Anal. 7, 434-448 (1961).

[10] Rajagopal, K. R., Wineman, A. S.: Developments in the mechanics of interactions between a fluid and a highly elastic solid. In: Recent developments in structured continua, Vol. II (DeKee, D., Kaloni, P. N., eds.), pp. 249-292. Pitman Research Notes in Mathematics Series, Longman Scientific and Technical, U. K. 1990.

[11] Rajagopal, K. R., Tao, L.: Mechanics of mixtures. Series on Advances in Mathematics for Applied Sciences vol. 35. Singapore: World Scientific 1995.

Authors' addresses: K. R. Rajagopal, Department of Mechanical Engineering, Texas A \& M University, College Station, Texas 77843, and A. Wineman, Department of Mechanical Engineering and Applied Mechanics, University of Michigan, Ann Arbor, Michigan 48109, U.S.A. 